\section{MP86-12 \\ PSYCHOLOGICAL DISTRESS IN PATIENTS UNDERGOING SURGERY FOR UROLOGICAL CANCER: A PROSPECTIVE SINGLE CENTRE CROSS-SECTIONAL STUDY}

Antonio Luigi Pastore*, Giovanni Palleschi, Antonio Carbone, Latina, Italy; Serena Maruccia, Milano, Italy; Ana Mir Bou, Nuria Camps Bellnoch, Juan Palou, Barcelona, Spain

INTRODUCTION AND OBJECTIVES: Interest in disease-specific psychological well-being of patients with cancer has increased, and it has been estimated that less than half of all cancer patients are properly identified and treated for anxiety or depression. The aim of this study was to evaluate psychological health assessment in oncological patients admitted for surgery.

METHODS: We performed a cross-sectional study in consecutively enrolled patients with bladder, kidney or prostate cancer scheduled for surgery. Demographic data, socioeconomic status, education level and diagnoses were recorded. We evaluated the level of clinically meaningful depression and anxiety assessed by two tools: the Hospital Anxiety and Depression Scale and the State-Trait Anxiety Inventory (STAI). In order to determine variables related to depression and anxiety among the demographic variables, logistic regression analyses were conducted, with $\mathrm{p}<0.05$ considered as statistically significant.

RESULTS: 207 patients completed the questionnaires and were included in the study. The most frequent procedures were performed for bladder tumours $(60.4 \%)$, being transurethral resection the most common type of surgery $(52.7 \%)$ followed by radical prostatectomy $(24.6 \%)$. The mean STAl-state score was $19.3( \pm 10.3)$, and the mean STAI-trait score was $18.4( \pm 11.9)$ points. Patients showed HADs depression and anxiety scores of $3.3( \pm 3)$ and $5.6( \pm 3.3)$ points, respectively. Female patients showed a higher level of anxiety and STAl-trait compared to males.

CONCLUSIONS: Gender, tumour type and surgical approach were significantly related to psychological distress in patients undergoing surgery for urological cancer. Females and patients with kidney tumour undergoing radical nephrectomy presented higher levels of anxiety.
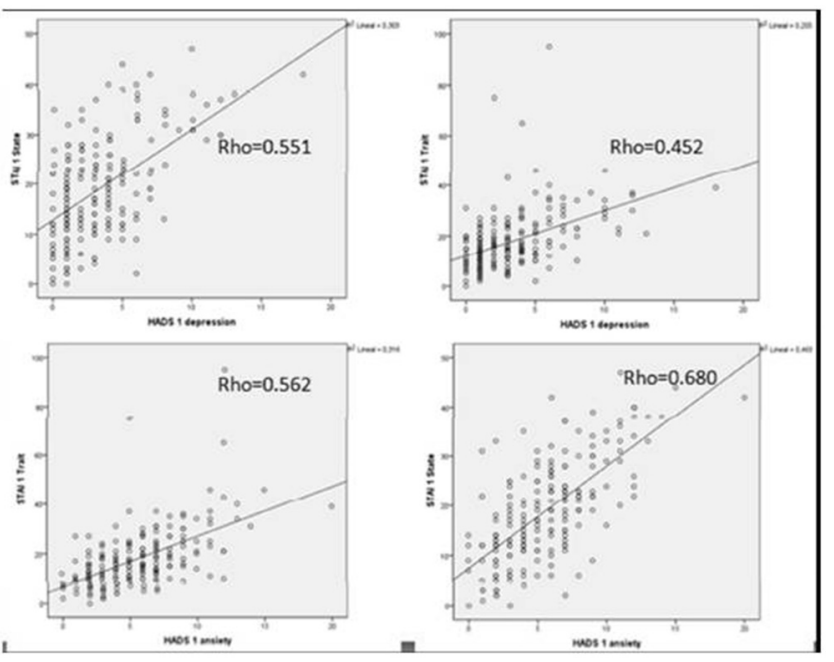

Source of Funding: None

\section{MP86-13 \\ HEALTH RELATED QUALITY OF LIFE OF PATIENTS UNDERGOING ACTIVE SURVEILLANCE - COMPARISON WITH PROSTATE CANCER ACTIVE TREATMENTS}

Lluis Fumado*, Barcelona, Spain; Jose Francisco Suárez, l'Hospitalet de Llobregat, Spain; Olatz Garín, Andrea Sureda, Montse Ferrer, Barcelona, Spain; Manel Castells, l'Hospitalet de Llobregat, Spain;
Jose Maria Abascal, Maria Carme Mir, Barcelona, Spain; Xavier Bonet, Helena Vila, l'Hospitalet de Llobregat, Spain; Lluís Cecchini, Barcelona, Spain; Francesc Vigués, l'Hospitalet de Llobregat, Spain

INTRODUCTION AND OBJECTIVES: Active surveillance in localised prostate cancer is an accepted option in selected cases, but some concerns still needs to be clarified. We pretend to evaluate Health-Related Quality of Life (HRQoL), in a cross-sectional study, in patients with localized prostate cancer undergoing active surveillance (AS) compared with active treatments (radical prostatectomy, externalbeam radiotherapy, brachytherapy) and general population.

METHODS: Ninety-nine patients fulfilled the inclusion criteria. This group was compared to different active treatments $(n=99$ per group) from the 'Spanish Multicentric Study of Clinically Localized Prostate Cancer' cohort using prostate-specific (Expanded Prostate Cancer Index Composite [EPIC]) and generic (36-Item Short Form Health Survey, version 2 [SF-36]) QoL instruments. SF-36 results were also compared to US reference population.

RESULTS: Cross-section analysis was performed at 24 months after initial therapy or AS beginning. AS group presented statistically higher sexual scores [54.4 mean score (28.3 SD)] when compared to all other active treatments and better urinary incontinence scores [87.4 (22.8 SD)] than radical prostatectomy group [65.8 (31.6 SD)]. Patients undergoing AS were more likely to present significant urinary irritative/ obstructive symptoms compared to radical prostatectomy group. No statistically significant differences were found among other domains. Patients with localized prostate cancer, regardless of treatment applied, presented slightly higher SF-36 physical and mental dimension scores (except for radical prostatectomy) than US general population reference norm (men aged 65-74)

CONCLUSIONS: AS may be a good treatment option for low or intermediate risk prostate cancer since induces the least impact in QoL. No significant differences were found on Physical and Mental Component Summaries compared to general US population. This study provides cross-sectional information about AS impact on QoL.

Source of Funding: This work was supported by grants from Instituto de Salud Carlos III FEDER (FIS PI11/01191 y FIS PI13/00412) and DIUE of Generalitat de Catalunya (2014 SGR 748)

\section{MP86-14 \\ PROSTATE SPECIFIC ANTIGEN AND HEALTH-RELATED QUALITY-OF-LIFE OUTCOMES IN UNINSURED MEN WITH PROSTATE CANCER}

Avi Baskin*, Joseph Shirk, Lorna Kwan, Karim Chamie, Los Angeles, $C A$

INTRODUCTION AND OBJECTIVES: Prostate Specific Antigen (PSA) continues to be a useful marker of risk strata and disease progression for patients with prostate cancer. While there have been multiple investigations into the relationship between PSA and mortality, there is a dearth in the literature describing the association between PSA and health-related quality-of-life (HRQOL) outcomes. In this study, we hypothesize that PSA is inversely related with $\mathrm{HRQOL}$ in patients with prostate cancer.

METHODS: Our cohort consisted of a prospective analysis of men from a state-funded program that provides free prostate cancer care to underinsured and uninsured low-income California residents with prostate cancer. Highest pre-treatment PSA was our primary exposure variable of interest. We created 4 strata: $<10,10-19.9,20-$ 49.9 and $\geq 50$ PSA. The primary outcome variables were HRQOL at program enrollment using the RAND SF-12 to measure physical and mental health, and the UCLA Prostate Cancer Index Short Form to measure urinary, sexual and bowel habits in two domains: bother and function. Controlling for demographic and clinical variables, we conducted separate multivariable linear regression analysis for each quality of life domain. 